\title{
A 2D-Covalent Organic Framework with Interlayer Hydrogen Bonding Oriented Through Designed Non-Planarity
}

Sampath B. Alahakoon, ${ }^{\dagger}$ Kui Tan, ${ }^{\S}$ Haardik Pandey ${ }^{\ddagger}$ Gregory T. McCandless, ${ }^{\dagger}$ Daniel I. Grinffiel, ${ }^{\dagger}$ Alejandra Durand-Silva, ${ }^{\dagger}$ Timo Thonhauser $^{\ddagger}$ and Ronald A. Smaldone ${ }^{\S, t^{*}}$

${ }^{\dagger}$ Department of Chemistry and Biochemistry, University of Texas at Dallas, 800 West Campbell Road, Richardson, Texas 75080, United States

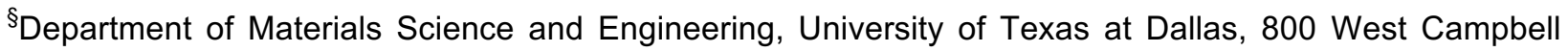
Road, Richardson, Texas 75080, United States

${ }^{\ddagger}$ Department of Physics and Center for Functional Materials, Wake Forest University, 1834 Wake Forest Road, Winston-Salem, North Carolina 27109, United States

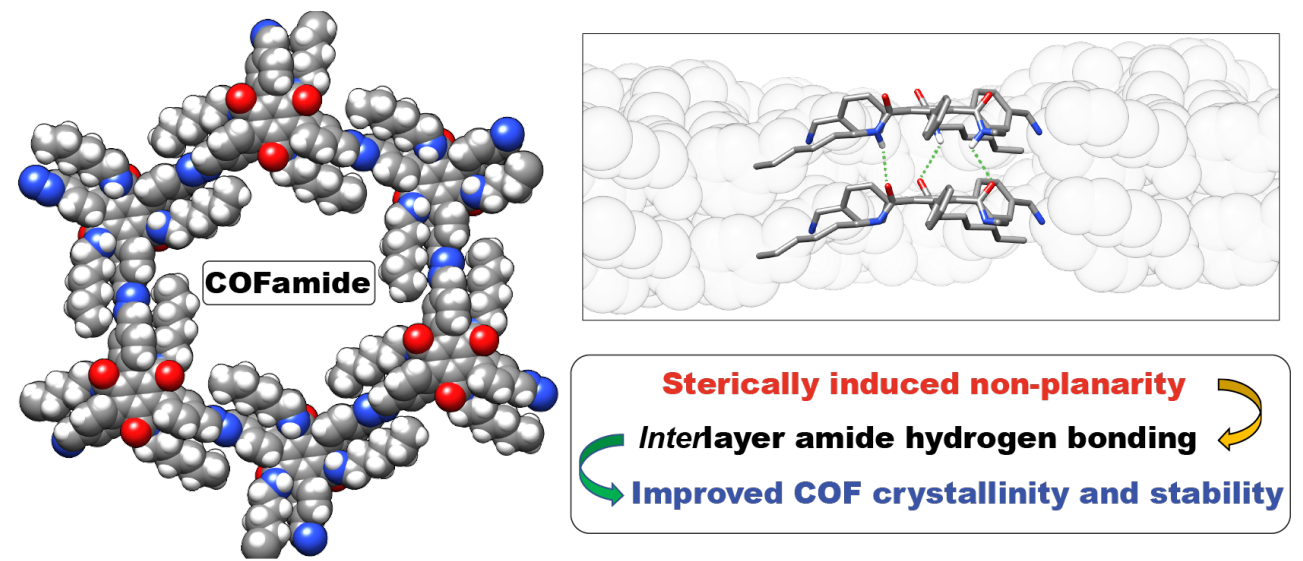

ABSTRACT: We report the synthesis and characterization of a new class of 2D-covalent organic frameworks, called COFamides, whose layers are held together by amide hydrogen bonds. To accomplish this, we have designed monomers with a non-planar structure that arises from steric crowding, forcing the amide side groups out of plane with the COF sheets orienting the hydrogen bonds between the layers. The presence of these hydrogen bonds provides significant structural stabilization as demonstrated by comparison to control structures that lack hydrogen bonding capability, resulting in lower surface area and crystallinity. We have characterized both azine and imine-linked versions of these COFs, named COFamide-1 and -2, respectively, for their surface areas, pore sizes and crystallinity. In addition to these more conventional characterization methods, we also used variable temperature infrared spectroscopy (VT-IR) methods and van der Waals density functional calculations to directly observe the presence of hydrogen bonding.

Introduction -2D-covalent organic frameworks (COFs) ${ }^{1}$ are a class of crystalline porous polymers comprised of covalently linked two-dimensional sheets which assemble through non-covalent interactions ${ }^{2-4}$ - typically aromatic stacking or dipolar interactions, generating a crystalline structure. The degree of relative crystallinity or pore fidelity can be changed by specifically tuning the non-covalent interactions between monomers through a variety of methods including modifying the electronic structure of the aromatic rings,${ }^{5-7}$ introducing donor-acceptor complexes, ${ }^{7}$ dipole cancellation between layers, ${ }^{8}$ and monomer planarity. ${ }^{9,10}$ However, despite the ubiquity of hydrogen bonding in self- 
assembled architectures in both Nature and synthetic supramolecular chemistry, there have been few reports of COFs that make use of hydrogen bonding between layers, ${ }^{11-14}$ though there have been many reports of COFs containing intralayer hydrogen bonds which serve to stabilize the dynamic imine bonds to hydrolysis. ${ }^{15,16}$

However, interlayer hydrogen bonding in COFs is much rarer. There are only a few examples of hydrazone ${ }^{17,18}$ or urea ${ }^{12}$ containing COFs where interlayer hydrogen bonding is observed or predicted, reported in literature. Several COFs have been reported that have interlayer $\mathrm{C}-\mathrm{H} \cdots \mathrm{O}$, and $\mathrm{C}-\mathrm{H} \cdots \mathrm{N}$ hydrogen bonding through sidechain interactions, ${ }^{13,14,19}$ however, more canonical $\mathrm{N}-\mathrm{H} \cdots \mathrm{O}$ hydrogen bonding interactions similar to those found between amide groups found in Nature, or in synthetic polymers such as Kevlar or Nylon, are much rarer.

Despite the difficulty in their preparation, there is a lot of potential for 2D-polymers with correlated hydrogen bonding between layers. Some reports have shown that hydrogen bonding can affect the photophysical ${ }^{17}$ or electrochemical ${ }^{13,14}$ properties of COFs. A recent theoretical study ${ }^{20}$ reported that 2D-materials, known as "graphamid" demonstrate mechanical properties exceeding those of para-aramid polymers such as Kevlar, owing to the amide hydrogen bonding between the layers. One of the most important structural features of graphamid is that the amide groups are forced out of plane with the aromatic rings by steric hindrance thereby orienting the hydrogen bonding interactions between layers. In practice, the synthesis of materials like graphamid is difficult due to the lack of dynamic reversibility in the formation of amide bonds as well as the preparation of monomers with the correct orientation of hydrogen bonding groups. As hydrogen bonding interactions are directional, the relative orientation between donor and acceptor is critical to their formation. Functional groups capable of acting as hydrogen bond donors or acceptors, such as amides or imines, tend to adopt a co-planar orientation with aromatic rings in order to remain in conjugation with the m-system. In 2D-COFs, this will typically result in hydrogen bonding interactions being oriented within the layers, rather than between them. 


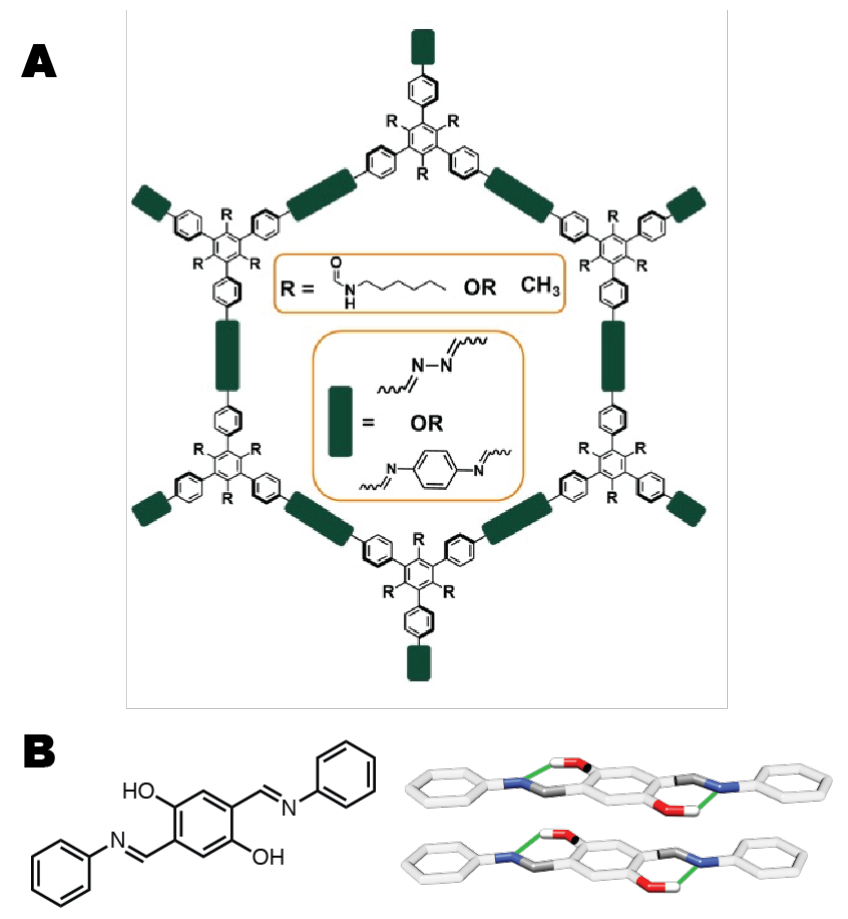

Intralayer hydrogen bonding

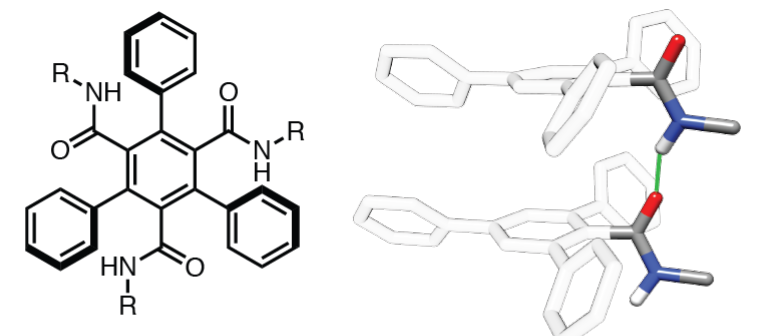

Interlayer hydrogen bonding

Figure 1. (A) Structure of the amide and control COFs linked through azine and imine bonds. (B) Co-planar hydrogen bonding functional groups will promote intralayer hydrogen bonding $(\mathrm{O}-\mathrm{H} \cdots \mathrm{N})$, whereas out of plane amides can form hydrogen bonding interactions between layers $(\mathrm{N}-\mathrm{H} \cdots \mathrm{O})$.

In order to create COFs capable of interlayer hydrogen bonding, we have designed a monomer containing secondary amides at the 1,3,5-positions of the central phenyl ring (Figure 1A). Since the 2,4,6-positions are substituted with phenyl groups, the amides adopt an out of plane conformation due to the steric bulk of the phenyl rings to facilitate interlayer hydrogen bonding during the polymer synthesis. Here the amide carbonyl oxygen of one layer will act as the hydrogen bond-acceptor while the amide hydrogen ( $\mathrm{N}$ $\mathrm{H}$ ) in the adjacent layer will act as the hydrogen bond-donor during the COF assembly (Figure 1B). This design was inspired by a previous report on self-assembled columnar liquid crystals derived from benzene-1,3,5-triamides. ${ }^{21}$ The amide containing COF 
monomer (1) was synthesized as described in Scheme S1. Hexylamides were used as they significantly improve the solubility of $\mathbf{1}$, which is beneficial not only for its purification, but also for the COF polymerization reaction as well.
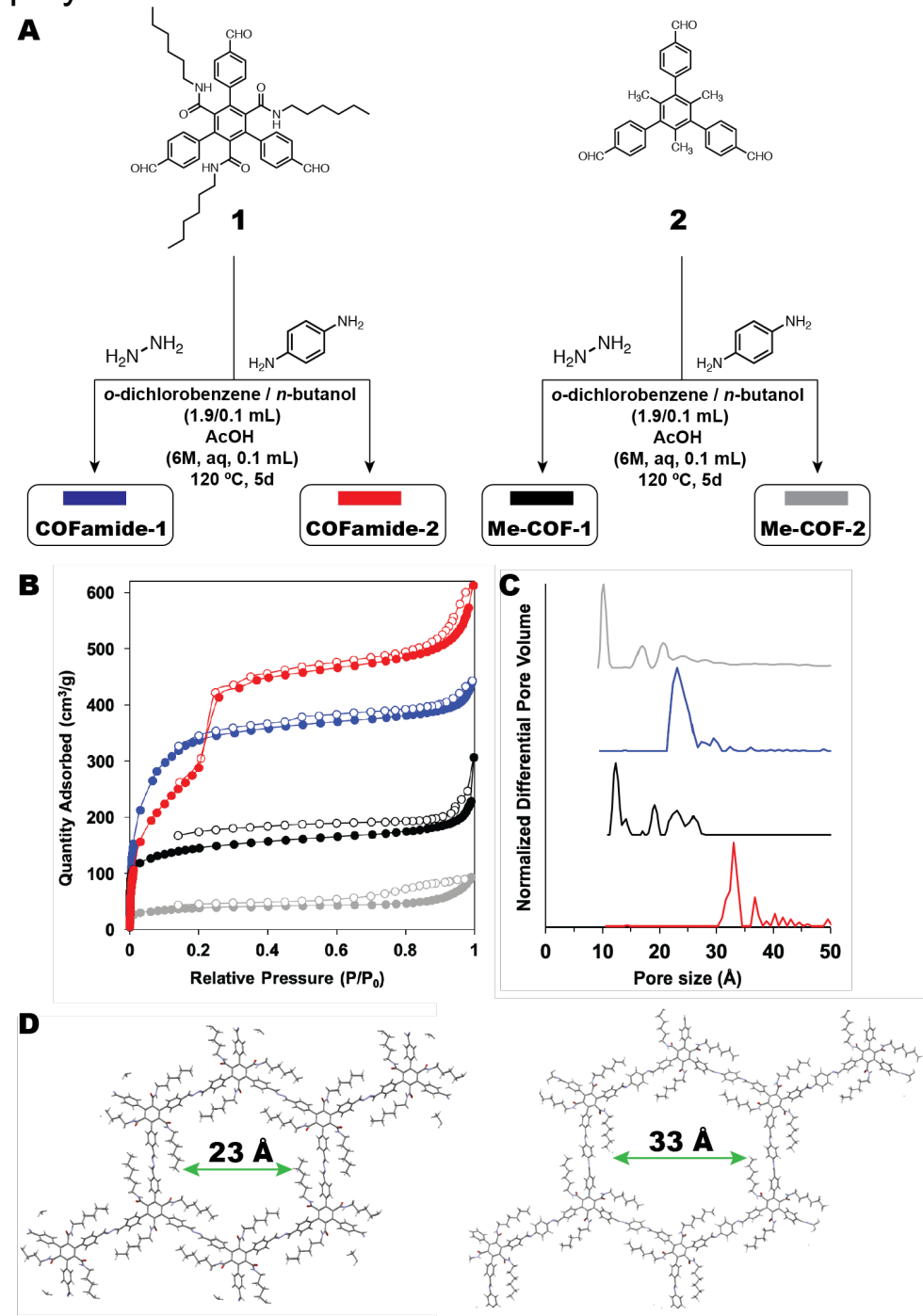

Cofamide-1

COFamide-2

Figure 2. (A) Synthesis of azine and imine COFs used in this study. (B) Nitrogen adsorption isotherms of all COFs, COFamide-1 (blue), COFamide-2 (red), Me-COF-1 (black), Me-COF-2 (grey). The filled circles are the adsorption isotherms and the open circles are desorption isotherms. (C) Non-local density functional theory (NLDFT) pore size distributions of all COFs. (D) Crystal structure models of COFamide-1 and -2, built in Materials Studio, with their predicted pore sizes. These values match closely with the measured NLDFT pore size distributions.

Monomer 1 was polymerized with both hydrazine and $p$-phenylenediamine to form the azine and imine-linked COFs, respectively (see also Scheme S2). These reactions were carried out in a solvent mixture of $o$-dichlorobenzene $(1.9 \mathrm{~mL})$ and $n$-butanol $(0.1 \mathrm{~mL})$ with acetic acid used as a catalyst $(6 \mathrm{M}(\mathrm{aq}), 0.1 \mathrm{~mL})$ for $5 \mathrm{~d}$ to yield the azine-linked COFamide- 
1 and imine-linked COFamide-2, respectively (Figure 2A). As a control, we have synthesized monomer 2 which is symmetrically analogous to 1 except the amide groups have been replaced with methyl groups that are incapable of hydrogen bonding. 2 was polymerized using the same conditions as monomer 1 . The formation of the azine and imine-linkages in COFamide-1 and -2 were confirmed by Fourier transform infrared spectroscopy spectra (FT-IR) through the attenuation of the aldehyde carbonyl stretching vibration $\left(1693 \mathrm{~cm}^{-1}\right)$ and the appearance of the azine $\left(1633 \mathrm{~cm}^{-1}\right)$ or imine $\left(1631 \mathrm{~cm}^{-1}\right)$ vibrations (Figure S1). For the control COFs, Me-COF-1 and Me-COF-2, the FT-IR spectra are shown in Figure S2.

Nitrogen adsorption measurements were carried out for the activated COFs to calculate the Brunauer-Emmett-Teller (BET) surface areas and the pore-size distributions using non-local density functional theory (NLDFT). These experiments revealed that both COFAmide-1 and 2 display type IV adsorption behavior which is characteristic in mesoporous materials. The BET surface areas of COFamide- 1 and 2 were measured to be 1271 and $1128 \mathrm{~m}^{2} / \mathrm{g}$, respectively (Figure 2B). This is more than double the surface areas obtained for the Me-COF-1 and -2 , of 536 and $138 \mathrm{~m}^{2} / \mathrm{g}$, respectively. The adsorption isotherms of these two COFs had shapes characteristic of type I isotherms indicating that they are microporous materials (Figure 2B). This measurement is inconsistent with the pore sizes predicted by crystal structure models (prepared in Materials Studio) of the Me-COFs if they formed crystalline structures with eclipsed structures, indicating that either the 2D-sheets are poorly or randomly aligned due to poor stacking, or have formed an amorphous 3D network rather than a layered 2D-COF structure. ${ }^{22,23} \mathrm{~S}$ In contrast, the experimental NLDFT pore size distributions of COFamide1 and 2 (Figure $2 \mathrm{C}$ ), are consistent with the pore sizes calculated from the simulated structures of $23 \AA$ and $32 \AA$, respectively (Figure 2D). This evidence supports the presence of an ordered structure consistent with the predicted models.
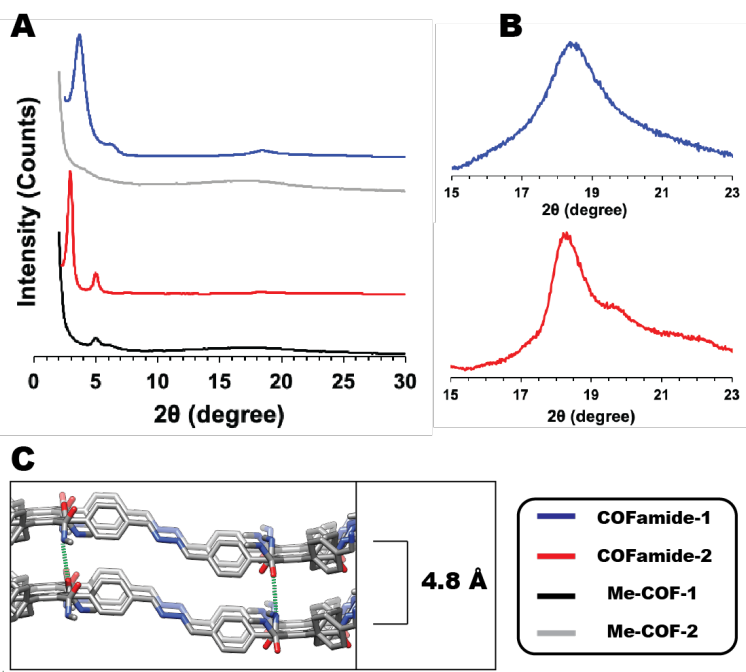

Figure 3. (A) PXRDs of all COFs. (B) Expanded region containing the 001 reflection of COFamide-1 and -2. (C) Side view of the COFamide-1 layers. The out of plane ring conformations forces the layers apart so that they have an interlayer distance of $4.8 \AA$ as calculated from the $2 \theta$ of the $001 \mathrm{hkl}$ reflection, larger than that of a typical COF held 
together by van der Waals, or m-stacking interactions (3.0-3.5 $\AA$ ). The locations of the expected hydrogen bonds are highlighted with green, dashed bonds. COFamide-1 (blue), COFamide-2 (red), Me-COF-1 (black), Me-COF-2 (grey).

The experimental determination of crystallinity of the COFs was carried out using powder X-ray diffraction (PXRD) measurements. The COFamide-1 and 2 displayed sharp diffraction peaks which can be attributed to their long-range order while Me-COF-1 and 2 were largely amorphous with only small low angle reflections (Figure $3 \mathrm{~A}$ ). We attribute the difference in bulk crystallinity of the amide and reference COFs to the improved layer stacking interactions that arise from the properly oriented amide hydrogen bonding between layers. Previous work has established that disruption of critical interlayer interactions can result in poor COF surface area, pore fidelity and overall crystallinity. ${ }^{24,25}$ Furthermore, we believe that the absence of these hydrogen bonds in Me-COF-1 and -2 results in their poor crystallinity. Out of plane phenyl ring torsions, or non-planar aromatic systems, have been attributed to both improved, ${ }^{26-28}$ and reduced ${ }^{9,10,29}$ crystallinity in COFs. Unless the non-planar monomer units can effectively "slip-stack" with one another, these torsions tend to act mostly as steric hindrance between the layers which reduces crystallinity and surface area. ${ }^{10,29}$ In the case of the amide functionalized COFs here, the out of plane torsions orient the $\mathrm{N}-\mathrm{H} \cdots \mathrm{O}$ hydrogen bonds between layers, which overcomes the steric hindrance seen in the Me-COFs, resulting in ordered COF materials when the amide functional groups are present.

Analysis of the powder X-ray diffraction patterns of these COFs further supports this hypothesis. Normally, the 001 reflection in a 2D-COF appears around $25^{\circ}$ and corresponds to the interlayer stacking distance $(3.0-3.5 \AA)$ where van der Waals and $\pi-$ stacking interactions are expected. Interestingly, this peak in COFAmide-1 and 2 appeared at around $18.5^{\circ}$ (Figure $3 \mathrm{~B}$ ). This reflection can be attributed to the increased interlayer distance which is $4.8 \AA$ as calculated using Bragg's Law. Models for both COFs were built in Materials Studio in the P6m space group (Figure S4, S10) and minimized in P1 using the universal force field (UFF). The simulated PXRDs of the eclipsed structure of both COFs match well with our experimental data indicating that COFAmide-1 and 2 are arranged in an eclipsed fashion rather than staggered (Figure S3, S9). These crystal structure models predict layer to layer stacking distances of $\sim 5.2 \AA$, similar to the experimental value $(\sim 4.8 \AA$ ) by PXRD (Figure $3 C$ ). These expanded layer stacking distances also provide more room for the amide hydrogen bonds to become properly oriented. We measured the $\mathrm{N}-\mathrm{H} \cdots \mathrm{O}$ distance (acceptor amide carbonyl oxygen of one layer to the donor amide hydrogen $(\mathrm{N}-\mathrm{H})$ of the adjacent layer) in the refined model and found them to be $\sim 2.6 \AA$, which is within the range of conventional hydrogen bonding (Figures S5, S11).

Spectroscopic Studies of COFs - Even though multiple experimental techniques including room temperature FT-IR analysis and solvent stability tests under harsh conditions have been previously reported to characterize interlayer hydrogen bonding in COFs, ${ }^{12,17-19}$ direct observation can still be challenging. We expected that the presence of strong hydrogen bonding interactions would lead to significant frequency shifts in IR spectrum of the functional groups directly involved in the hydrogen-bonded bridges. In 
order to directly observe these hydrogen bonding interactions, we performed variable temperature FT-IR experiments (VT-IR, Figure 4). In previous spectroscopic studies of amide containing polymers ${ }^{30-32}$ the hydrogen bonding is disrupted at elevated temperatures resulting in the amide carbonyl and amide $\mathrm{N}-\mathrm{H}$ bond stretching frequencies blue shifting due to the increase in bond orders of the carbonyl and $\mathrm{N}-\mathrm{H}$ bonds.
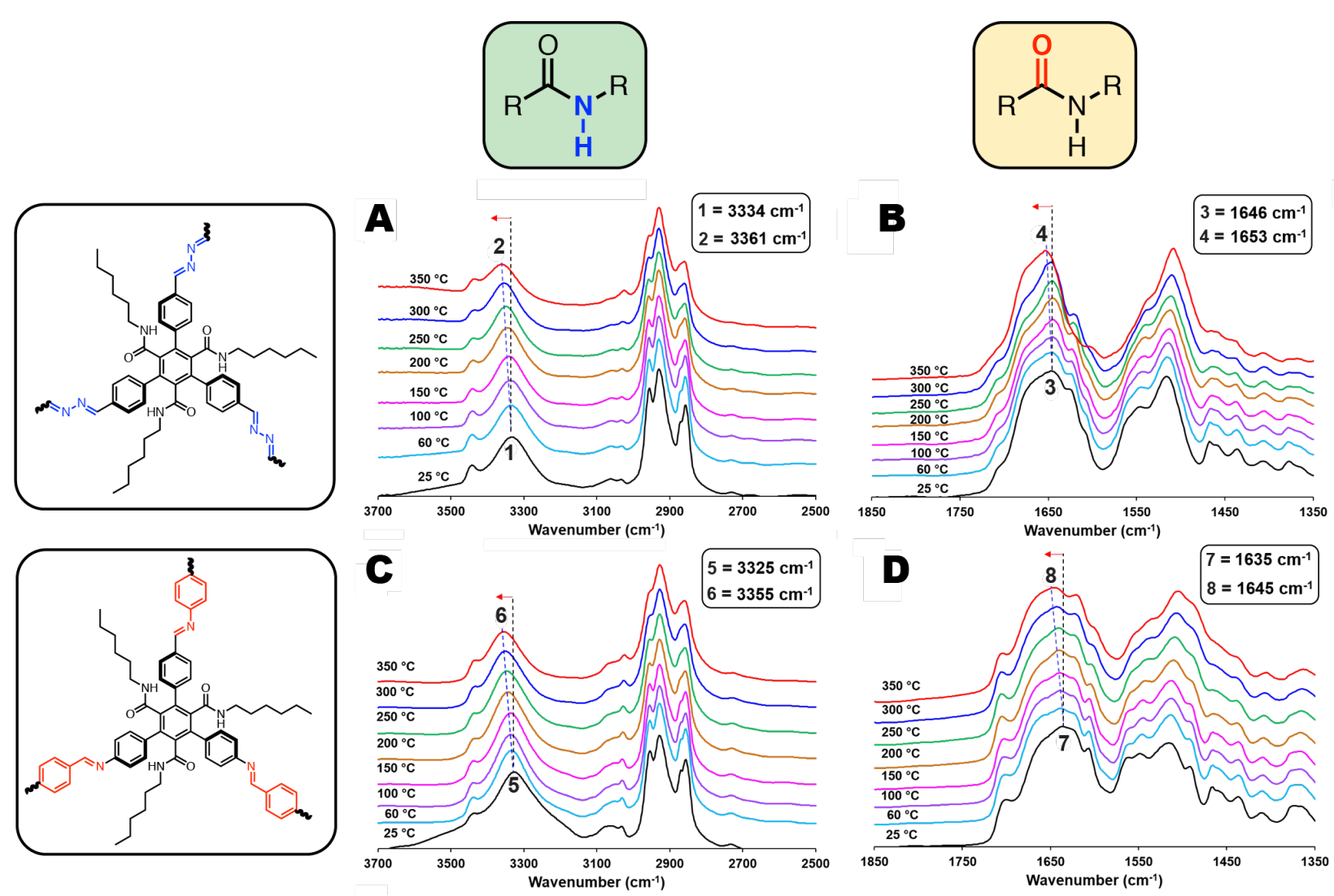

Figure 4. Variable temperature $I R$ spectra of COFamide-1 and -2. COFAmide-1: (A) Expanded amide N-H stretching region and (B) Zoomed in amide carbonyl stretching region. COFAmide-2: (C) Expanded amide N-H stretching region and (D) Zoomed in amide carbonyl stretching region.

In each VT-IR experiment, spectra were collected at temperatures ranging from room temperature up to $350{ }^{\circ} \mathrm{C}$. Upon heating the sample, the vibrational frequencies that correspond to amide $\mathrm{N}-\mathrm{H}$ and amide carbonyl started shifting towards the higher wavenumber region (Figure $4 A$ and $B$, respectively) with no effect on the other vibrational peaks in the spectrum. In COFamide-1, the amide N-H vibrational peak appears at 3334 $\mathrm{cm}^{-1}$ at room temperature. When the sample heated to $350^{\circ} \mathrm{C}$ the peak moved gradually to $3361 \mathrm{~cm}^{-1}$ with an overall shift of $27 \mathrm{~cm}^{-1}$ (Figure 4A). This shift upon heating can be attributed to the disruption of the hydrogen bonding and an increase in the $\mathrm{N}-\mathrm{H}$ bond order because of the formation of free $\mathrm{N}-\mathrm{H}$ sites. The amide carbonyl vibration peak at room temperature appears at $1646 \mathrm{~cm}^{-1}$, while it gradually shifts to $1653 \mathrm{~cm}^{-1}$ with an overall shift of $7 \mathrm{~cm}^{-1}$ when heated to $350^{\circ} \mathrm{C}$ (Figure 4B). This also can be attributed to the increasing bond order due to the formation of free amide carbonyls with increasing 
temperature. In COFamide-2 similar vibrational shifts were observed as of COFamide-1 (Figure $4 \mathrm{C}$ and $\mathrm{D}$ ). These observations are consistent with the previous variable temperature IR studies on polyamides. ${ }^{30-32}$

Computational Studies - Though the simulated crystal structure models and PXRD data support the presence of hydrogen bonding, we sought to obtain atomic scale information about the hydrogen bonding through theoretical calculations. To accomplish this, we employed density functional theory (DFT) calculations using the van der Waals density functional (vdW-DF) that is capable of accurately representing intermolecular interactions such as hydrogen bonding.

The COF structure was reduced to a repeating fragment that consisted of two 2dimensional layers with hydrogen termination where necessary (Figure 5A). The structure was properly periodic in the direction perpendicular to the layers and contained a total of 144 atoms in the repeat unit cell. Ab initio calculations were performed at the DFT level, using VASP ${ }^{33,34}$ in conjunction with the vdW-DF method. ${ }^{35-38}$ The plane-wave energy cutoff was set at $600 \mathrm{eV}$ and the SCF convergence condition was set to be $10^{-3} \mathrm{meV}$. To get an optimal separation between the layers of the COF, the lattice parameter was allowed to change in the direction perpendicular to the plane of the 2D-layers. The structure was optimized until all forces between atoms were smaller than $1 \mathrm{meV} / \AA$. The total energy of the optimized 2-layer structure and single-layer structures were used to calculate the binding energy between the layers. Induced charge densities were calculated as the difference between the fully self-consistent density of the 2-layer system minus the corresponding single-layer densities. Induced charge densities thus show the rearrangement of charge density upon bringing the layers together and help identifying the formation of bonds and/or interactions.

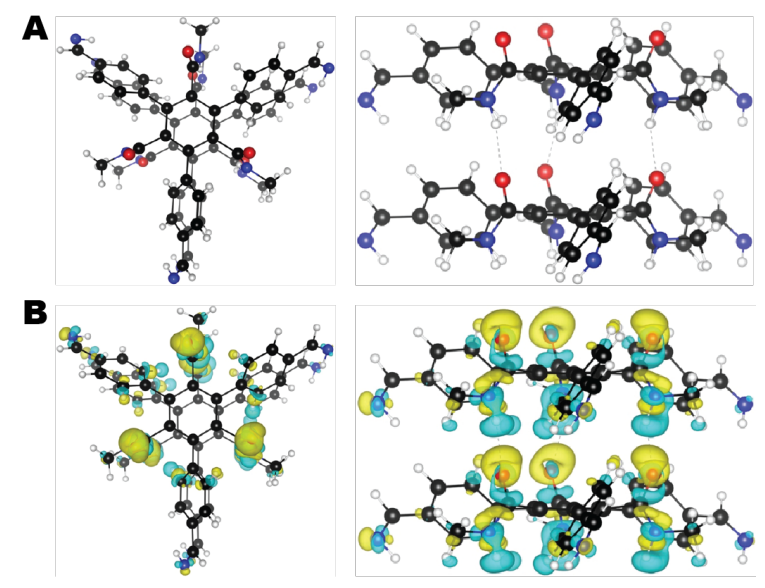

Figure 5. (A) Computational model of stacked molecules of monomer 1 with hydrogen bonding depicted by dotted lines. (B) Induced charge densities at an iso-level of 0.0015 electrons $/ \AA^{3}$. Yellow areas represent a charge accumulation and blue areas a depletion upon bringing the layers together. Three "chains" of induced charge per fragment become visible, showing the hydrogen bonds that link the layers together. 
The optimized COF structure had an interplanar distance of $5.03 \AA$, when measured from the benzene molecule in one layer to the benzene in another. This is similar to the $4.8 \AA$ measured experimentally, and the $5.2 \AA$ distance from the molecular mechanics based crystal structure calculations performed in Materials Studio using the universal force field (UFF). The binding energy between the two layers of the fragments depicted in Figure $5 \mathrm{~A}$ was calculated to be $152.97 \mathrm{~kJ} / \mathrm{mol}(1.58 \mathrm{eV})$, resulting in a binding energy per unit area of $0.47 \mathrm{~kJ} / \mathrm{mol} / \AA^{2}\left(4.49 \mathrm{meV} / \AA^{2}\right)$ for the entire COF structure. We calculated the $\mathrm{N}$ $\mathrm{H} \cdots \mathrm{O}$ hydrogen bonding distance to be $2.09 \AA$. While is smaller than the value from our simulated crystal structure, it is still well within the range of a canonical hydrogen bonding interaction.

Induced charge densities are shown in Figure 5B. The plot shows an increase in electron density at the oxygen atoms and a decrease around hydrogen atoms-a clear indication of a hydrogen bonding interaction. We find most of the density change concentrated along the functional groups containing the hydrogen binding atoms. The induced charge densities along with the bond distance and binding energy show that the layers of the COF are more strongly held together by the $\mathrm{N}-\mathrm{H} \cdots \mathrm{O}$ hydrogen bonds.

Conclusions - In summary, we were able to design and synthesize a COF with an extended network of hydrogen bonding through amide functional groups. Due to steric crowding, the amide units adopt an out of plane conformation which promotes interlayer (rather than intralayer) hydrogen bonding between pendant amide groups when assembled into a crystalline framework. When compared to a control COF without amide groups, COFAmide-1 and -2 display significantly improved material properties such as bulk crystallinity and surface area. These improved material properties were attributed to the 2D assembly directed through strong interlayer hydrogen bonding. We have also demonstrated the value of VT-IR as a tool to experimentally observe the existence of hydrogen bonding between layers in a COF. From a practical perspective, amide hydrogen bonding is important for mechanical strength in polymeric materials, therefore reliable, and designable methods to incorporate these interactions in COFs could help expand them into new applications. We also see this design strategy as a general method that will allow for the synthesis of new COF materials. Furthermore, this will enable monomers that are too sterically bulky to interface with one another to be effectively incorporated into 2D-COFs by independently stabilizing the eclipsed stacking mode.

\section{ACKNOWLEDGMENTS}

R.A.S acknowledges support from the Army Research Laboratory (W911NF-18-2-0035). The vibrational spectroscopy and DFT simulations were supported by the U.S. Department of Energy, Basic Energy Sciences, Division of Materials Sciences and Engineering, Grant No. DE-SC0019902 (K.T., T.T.). A.D.S acknowledges Consejo Nacional de Ciencia y Tecnología (National Council for Science and Technology) of Mexico for a doctoral fellowship. 


\section{REFERENCES}

(1) Côté, A. P.; Benin, A. I.; Ockwig, N. W.; Keeffe, M.; Matzger, A. J.; Yaghi, O. M. "Porous, Crystalline, Covalent Organic Frameworks," Science 2005, 310, 1166.

(2) Colson, J. W.; Dichtel, W. R. "Rationally Synthesized Two-Dimensional Polymers," Nat. Chem. 2013, 5, 453-465.

(3) Ding, S.-Y.; Wang, W. "Covalent Organic Frameworks (COFs): From Design to Applications," Chem. Soc. Rev. 2013, 42, 548-568.

(4) Alahakoon, S. B.; Diwakara, S. D.; Thompson, C. M.; Smaldone, R. A. "Supramolecular Design in 2D Covalent Organic Frameworks," Chem. Soc. Rev. 2020, 49, 1344-1356.

(5) Alahakoon, S. B.; McCandless, G. T.; Karunathilake, A. A. K.; Thompson, C. M.; Smaldone, R. A. "Enhanced Structural Organization in Covalent Organic Frameworks through Fluorination," Chem. Eur. J. 2017, 23, 4255-4259.

(6) Alahakoon, S. B.; Occhialini, G.; McCandless, G. T.; Karunathilake, A. A. K.; Nielsen, S. O.; Smaldone, R. A. "Experimental and Theoretical Insight into the Effect of Fluorine Substituents on the Properties of Azine Linked Covalent Organic Frameworks," CrystEngComm 2017.

(7) Chen, X.; Addicoat, M.; Irle, S.; Nagai, A.; Jiang, D. "Control of Crystallinity and Porosity of Covalent Organic Frameworks by Managing Interlayer Interactions Based on Self-Complementary m-Electronic Force," J. Am. Chem. Soc. 2013, 135, 546-549.

(8) Salonen, L. M.; Medina, D. D.; Carbó-Argibay, E.; Goesten, M. G.; Mafra, L.; Guldris, N.; Rotter, J. M.; Stroppa, D. G.; Rodríguez-Abreu, C. "A Supramolecular Strategy Based on Molecular Dipole Moments for High-Quality Covalent Organic Frameworks," Chem. Commun. 2016, 52, 7986-7989.

(9) Vyas, V. S.; Haase, F.; Stegbauer, L.; Savasci, G.; Podjaski, F.; Ochsenfeld, C.; Lotsch, B. V. "A Tunable Azine Covalent Organic Framework Platform for Visible Light-Induced Hydrogen Generation," Nat. Commun. 2015, 6, 8508.

(10) Thompson, C. M.; Occhialini, G.; McCandless, G. T.; Alahakoon, S. B.; Cameron, V.; Nielsen, S. O.; Smaldone, R. A. "Computational and Experimental Studies on the Effects of Monomer Planarity on Covalent Organic Framework Formation," J. Am. Chem. Soc. 2017, 139, 10506-10513. 
(11) Halder, A.; Karak, S.; Addicoat, M.; Bera, S.; Chakraborty, A.; Kunjattu, S. H.; Pachfule, P.; Heine, T.; Banerjee, R. "Ultrastable Imine-Based Covalent Organic Frameworks for Sulfuric Acid Recovery: An Effect of Interlayer Hydrogen Bonding," Angew. Chem. Int. Ed. 2018, 57, 5797-5802.

(12) Zhao, C.; Diercks, C. S.; Zhu, C.; Hanikel, N.; Pei, X.; Yaghi, O. M. "Urea-Linked Covalent Organic Frameworks," J. Am. Chem. Soc. 2018, 140, 16438-16441.

(13) Halder, A.; Ghosh, M.; Khayum M, A.; Bera, S.; Addicoat, M.; Sasmal, H. S.; Karak, S.; Kurungot, S.; Banerjee, R. "Interlayer Hydrogen-Bonded Covalent Organic Frameworks as High-Performance Supercapacitors," J. Am. Chem. Soc. 2018, 140, 10941-10945.

(14) Li, L.; Lu, F.; Xue, R.; Ma, B.; Li, Q.; Wu, N.; Liu, H.; Yao, W.; Guo, H.; Yang, W. "Ultrastable Triazine-Based Covalent Organic Framework with an Interlayer Hydrogen Bonding for Supercapacitor Applications," ACS Appl. Mater. Interfaces 2019, 11, 26355-26363.

(15) Kandambeth, S.; Shinde, D. B.; Panda, M. K.; Lukose, B.; Heine, T.; Banerjee, R. "Enhancement of Chemical Stability and Crystallinity in Porphyrin-Containing Covalent Organic Frameworks by Intramolecular Hydrogen Bonds," Angew. Chem. Int. Ed. 2013, 52, 13052-13056.

(16) Mo, Y.-P.; Liu, X.-H.; Sun, B.; Yan, H.-J.; Wang, D.; Wan, L.-J. "The Intramolecular $\mathrm{H}$-Bonding Effect on the Growth and Stability of Schiff-Base Surface Covalent Organic Frameworks," Phys. Chem. Chem. Phys. 2017, 19, 539-543.

(17) Li, X.; Gao, Q.; Wang, J.; Chen, Y.; Chen, Z.-H.; Xu, H.-S.; Tang, W.; Leng, K.; Ning, G.-H.; Wu, J.; Xu, Q.-H.; Quek, S. Y.; Lu, Y.; Loh, K. P. "Tuneable near White-Emissive Two-Dimensional Covalent Organic Frameworks," Nat. Commun. 2018, 9, 2335.

(18) Li, X.; Qiao, J.; Chee, S. W.; Xu, H.-S.; Zhao, X.; Choi, H. S.; Yu, W.; Quek, S. Y.; Mirsaidov, U.; Loh, K. P. "Rapid, Scalable Construction of Highly Crystalline Acylhydrazone Two-Dimensional Covalent Organic Frameworks via DipoleInduced Antiparallel Stacking," J. Am. Chem. Soc. 2020, 142, 4932-4943.

(19) Halder, A.; Kandambeth, S.; Biswal, B. P.; Kaur, G.; Roy, N. C.; Addicoat, M.; Salunke, J. K.; Banerjee, S.; Vanka, K.; Heine, T.; Verma, S.; Banerjee, R. "Decoding the Morphological Diversity in Two Dimensional Crystalline Porous Polymers by Core Planarity Modulation," Angew. Chem. Int. Ed. 2016, 55, 78067810.

(20) Sandoz-Rosado, E.; Beaudet, T. D.; Andzelm, J. W.; Wetzel, E. D. "High Strength Films from Oriented, Hydrogen-Bonded "Graphamid" 2D Polymer Molecular Ensembles," Sci. Rep. 2018, 8, 3708. 
(21) Bushey, M. L.; Nguyen, T.-Q.; Zhang, W.; Horoszewski, D.; Nuckolls, C. "Using Hydrogen Bonds to Direct the Assembly of Crowded Aromatics," Angew. Chem. Int. Ed. 2004, 43, 5446-5453.

(22) Sick, T.; Rotter, J. M.; Reuter, S.; Kandambeth, S.; Bach, N. N.; Döblinger, M.; Merz, J.; Clark, T.; Marder, T. B.; Bein, T.; Medina, D. D. "Switching on and Off Interlayer Correlations and Porosity in 2D Covalent Organic Frameworks," J. Am. Chem. Soc. 2019, 141, 12570-12581.

(23) Feriante, C. H.; Jhulki, S.; Evans, A. M.; Dasari, R. R.; Slicker, K.; Dichtel, W. R.; Marder, S. R. "Rapid Synthesis of High Surface Area Imine-Linked 2D Covalent Organic Frameworks by Avoiding Pore Collapse During Isolation," Adv. Mater. 2020, 32, 1905776.

(24) Spitler, E. L.; Koo, B. T.; Novotney, J. L.; Colson, J. W.; Uribe-Romo, F. J.; Gutierrez, G. D.; Clancy, P.; Dichtel, W. R. "A 2D Covalent Organic Framework with 4.7-Nm Pores and Insight into Its Interlayer Stacking," J. Am. Chem. Soc. 2011, 133, 19416-19421.

(25) Alahakoon, S. B.; Thompson, C. M.; Occhialini, G.; Smaldone, R. A. "Design Principles for Covalent Organic Frameworks in Energy Storage Applications," ChemSusChem 2017, 10, 2116-2129.

(26) Ascherl, L.; Sick, T.; Margraf, J. T.; Lapidus, S. H.; Calik, M.; Hettstedt, C.; Karaghiosoff, K.; Döblinger, M.; Clark, T.; Chapman, K. W.; Auras, F.; Bein, T. "Molecular Docking Sites Designed for the Generation of Highly Crystalline Covalent Organic Frameworks," Nat. Chem. 2016, 8, 310-316.

(27) Auras, F.; Ascherl, L.; Hakimioun, A. H.; Margraf, J. T.; Hanusch, F. C.; Reuter, S.; Bessinger, D.; Döblinger, M.; Hettstedt, C.; Karaghiosoff, K.; Herbert, S.; Knochel, P.; Clark, T.; Bein, T. "Synchronized Offset Stacking: A Concept for Growing Large-Domain and Highly Crystalline 2D Covalent Organic Frameworks," J. Am. Chem. Soc. 2016, 138, 16703-16710.

(28) Martínez-Abadía, M.; Stoppiello, C. T.; Strutynski, K.; Lerma-Berlanga, B.; MartíGastaldo, C.; Saeki, A.; Melle-Franco, M.; Khlobystov, A. N.; Mateo-Alonso, A. "A Wavy Two-Dimensional Covalent Organic Framework from Core-Twisted Polycyclic Aromatic Hydrocarbons," J. Am. Chem. Soc. 2019, 141, 14403-14410.

(29) Alahakoon, S. B.; Thompson, C. M.; Nguyen, A. X.; Occhialini, G.; McCandless, G. T.; Smaldone, R. A. "An Azine-Linked Hexaphenylbenzene Based Covalent Organic Framework," Chem. Commun. 2016, 52, 2843-2845. 
(30) Skrovanek, D. J.; Howe, S. E.; Painter, P. C.; Coleman, M. M. "Hydrogen Bonding in Polymers: Infrared Temperature Studies of an Amorphous Polyamide," Macromolecules 1985, 18, 1676-1683.

(31) Coleman, M. M.; Skrovanek, D. J.; Painter, P. C. "Hydrogen-Bonding in Polymers. 3. Further Infrared Temperature Studies of Polyamides.," Makromol. Chemie. Macromol. Symp. 1986, 5, 21-33.

(32) Skrovanek, D. J.; Painter, P. C.; Coleman, M. M. "Hydrogen Bonding in Polymers. 2. Infrared Temperature Studies of Nylon 11," Macromolecules 1986, 19, 699-705.

(33) Kresse, G.; Furthmüller, J. "Efficient Iterative Schemes for ab initio Total-Energy Calculations Using a Plane-Wave Basis Set," Phys. Rev. B 1996, 54, 1116911186.

(34) Kresse, G.; Joubert, D. "From Ultrasoft Pseudopotentials to the Projector Augmented-Wave Method," Phys. Rev. B 1999, 59, 1758-1775.

(35) Thonhauser, T.; Cooper, V. R.; Li, S.; Puzder, A.; Hyldgaard, P.; Langreth, D. C. "van der Waals Density Functional: Self-Consistent Potential and the Nature of the van der Waals Bond," Phys. Rev. B 2007, 76, 125112.

(36) Langreth, D. C.; Lundqvist, B. I.; Chakarova-Käck, S. D.; Cooper, V. R.; Dion, M.; Hyldgaard, P.; Kelkkanen, A.; Kleis, J.; Kong, L.; Li, S.; Moses, P. G.; Murray, E.; Puzder, A.; Rydberg, H.; Schröder, E.; Thonhauser, T. "A Density Functional for Sparse Matter," J. Phys. Condens. Matter 2009, 21, 084203.

(37) Berland, K.; Cooper, V. R.; Lee, K.; Schröder, E.; Thonhauser, T.; Hyldgaard, P.; Lundqvist, B. I. "van der Waals Forces in Density Functional Theory: A Review of the vdW-DF Method," Reports Prog. Phys. 2015, 78, 066501.

(38) Thonhauser, T.; Zuluaga, S.; Arter, C. A.; Berland, K.; Schröder, E.; Hyldgaard, P. "Spin Signature of Nonlocal Correlation Binding in Metal-Organic Frameworks," Phys. Rev. Lett. 2015, 115, 136402. 